Impulsive Start of a Symmetric Airfoil at High Angle of Attack Joseph Katz, Steven Yon, Stuart E. Rogers

Reprinted from

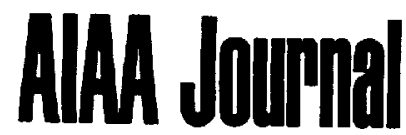

Volume 34, Number 2, Pages 225-230

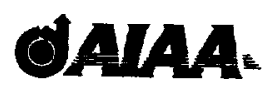

A publication of the

American Institute of Aeronautics and Astronautics, Inc.

370 L'Enfant Promenade, SW

Washington, DC 20024-2518 



\title{
Impulsive Start of a Symmetric Airfoil at High Angle of Attack
}

\author{
Joseph Katz* and Steven Yon ${ }^{\dagger}$ \\ San Diego State University, San Diego, California 92182-0183 \\ and \\ Stuart E. Rogers ${ }^{\ddagger}$ \\ NASA Ames Research Center, Moffett Field, Califomia 94035-1000
}

\begin{abstract}
The fluid dynamic phenomena following the impulsive start of a NACA 0015 airfoil were studied by using a time aceurate solution of the incompressible, laminar Navier-Stokes equations. Angle of attack was set at 10 deg to simulate steady-state poststall conditions at a Reynolds number of $1.2 \times 10^{4}$. The calculation revealed that large initial lift values can be obtained, immediately following the impulsive start, when a trapped vortex develops above the airfoil. Before the buildup of this trapped vortex and immediately after the airfoil was set into motion, the fluid is attached to the airfoil's surface and flows around the trailing edge, demonstrating the delay in the buildup of the classical Kutta condition. The transient of this effect is quite short and is followed by an attached flow event that leads to the trapped vortex that has a longer duration. The just described initial phenomenon eventually transits into a fully developed separated flow pattern identifiable by an alternating, periodic vortex shedding.
\end{abstract}

\section{Introduction}

$\mathbf{T}$ HE impulsive start of an airfoil at an angle of attack, conceptually, is one of the simplest examples of unsteady aerodynamics. This assumption is based on the logic that a step function results in the simplest and most basic time-dependent response for a given system. However, the impulsive start of an airfoil is far from being one of the simplest unsteady fluid dynamic examples. For instance, the experimental evaluation of the fluid dynamic quantities must properly separate the fluid dynamic loads from the inertia of the support system, which is probably the reason why there are very little data published on this problem. One of the earliest and most basic analytical studies about a step change in the forward velocity of an airfoil was published by Wagner ${ }^{1}$ in 1925 . This first attempt was based on a thin-wing, potential-flow model and was able to demonstrate the delay in the buildup of lift, due to the downwash of the starting vortex. This model of Ref. 1 was gradually improved to include effects of thickness and of flexibility. ${ }^{2-5}$ Similar ideal flow models for the higher angles of attack range followed, and Ref. 6 presents an example for such a model, whereas Refs. 7 and 8 demonstrate the extension of this model into three dimensions.

Experimental results (e.g., Refs. 9-12) on this basic problem are quite scarce, as mentioned earlier, primarily because of the technical difficulties involved in this seemingly simple experiment. Part of the problem lies in the inertial effects of both airfoil model and surrounding fluid during the initial acceleration that cause various time delays in the measured data. Therefore, a logical and costeffective approach to study this very basic example of unsteady fluid dynamics is by solving the viscous-flow equations in a time accurate manner.

\section{Method of Solution}

The computer code, INS2D, ${ }^{13-15}$ was used to solve the twodimensional incompressible Navier-Stokes equations for the flow over the airfoil. This code was developed to solve both steady-state and time-dependent problems. The INS2D code has been validated

Received April 17, 1995; presented as Paper 95-1872 at the AIAA 13th Applied Aerodynamics Conference, San Diego, CA, June 19-22, 1995; revision received Aug. 22, 1995; accepted for publication Aug. 25, 1995. Copyright (C) 1995 by the American Institute of Aeronautics and Astronautics, Inc. All rights reserved.

"Professor, Department of Aerospace Engineering and Engineering Mechanics. Associate Felow AIAA.

${ }^{\dagger}$ Graduate Student, Department of Aerospace Engineering and Engineering Mechanics. Student Member AIAA.

${ }^{*}$ Aerospace Engineer, Design Cycle Technologies Branch. Member AIAA. and used extensively to simulate steady-state high Reynolds number flow over airfoils, ${ }^{16.17}$ as well unsteady flows with airfoils. ${ }^{18}$ The code uses the method of artificial compressibility; for timedependent solutions this requires the use of subiterations at each physical time step to solve for a (nearly) divergence-free velocity field. In the code, the convective terms are differenced using a thirdorder accurate upwind-biased flux-difference splitting method, the viscous terms are differenced using a second-order central difference method, and the equations are integrated in time using a secondorder scheme. The implicit solution uses an iterative, generalizedminimum-residual matrix solver resulting in fast convergence for steady-state problems and the ability to use large time steps for time-dependent simulations.

The present study focuses on the impulsive start of the airfoil within the laminar flow range only. The Reynolds number based on the airfoil chord $c$ and the freestream velocity $U_{\infty}$ was set at $1.2 \times 10^{4}$; this value matches the conditions of a water-tunnel validation experiment planned for the future. At this low Reynolds number, unknowns associated with the modeling of turbulence are avoided, leaving only the effects relevant to the impulsive start. However, features such as the vortex wake roll-up can be related to flows with higher Reynolds number. Based on this hypothesis, therefore, the dominant flow features following the airfoil's impulsive start will remain in the higher Reynolds number flow case, but the airfoil's stall will be delayed to higher angles of attack.

A C-grid of dimensions $401 \times 121$ was used in this study, and Fig. 1 depicts the grid in the vicinity of the NACA 0015 airfoil. The higher density cells near the airfoil surface were obtained by using a single-block hyperbolic grid generator. A grid resolution study using grid densities of $401 \times 121,201 \times 61$, and $101 \times 31$

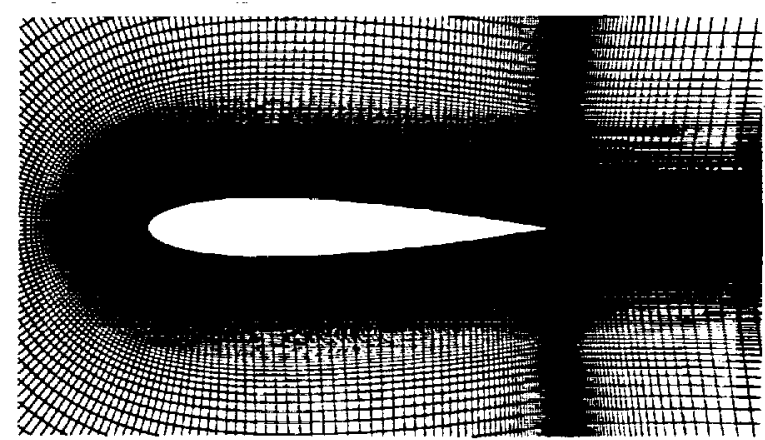

Fig. 1 Finite difference grid $(401 \times 121)$ around a NACA 0015 airfoil. 


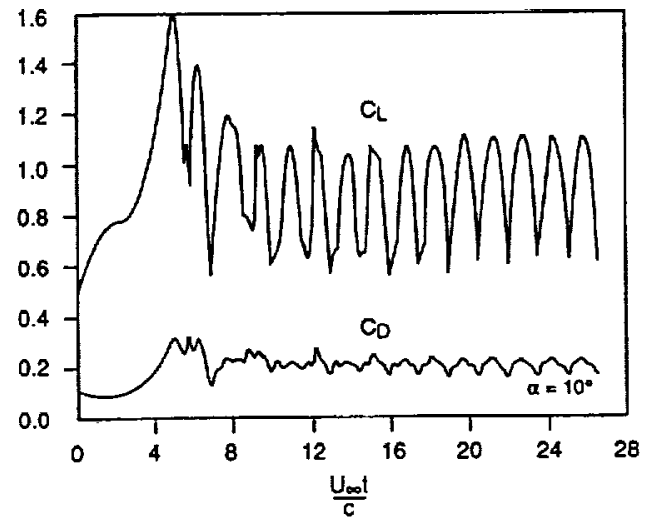

Fig. 2 Lift and drag coefficients vs time for the NACA 0015 airfoil at an angle of attack of $10 \mathrm{deg}$ and time step of $\Delta t=U_{\infty} t / c=0.025$.

showed that the two finest grids produced nearly the same results, but that it was necessary to use the $401 \times 121$ grid to resolve the vortex features found in this flowfield. Numerical tests of the effect of the nondimensional time step $U_{\infty} \Delta t / c$ used in the code were done using values of $0.0125,0.025$, and 0.05 . It was found that the solutions for values of 0.0125 and 0.025 were identical, and thus the latter value was used for all computations except during the initial startup period. During the initial startup of this impulsive motion $\left(0.0<U_{\infty} t / c<0.02\right)$, a much smaller time step was necessary. Numerical tests showed that a nondimensional time step of 0.0005 was adequate for this time interval. Also, on the airfoil surface the no-slip boundary condition and on the far-field boundaries a uniform freestream condition were imposed (except at the outflow boundary where an updating scheme was used).

The airfoil section angle of attack was set at $10 \mathrm{deg}$, a condition that is slightly above stall for this particular Reynolds number. This borderline condition allowed the investigation of the possibility to increase an airfoil's lift on the verge of flow separation. Also, this condition can provide information about the time delay between the momentary attached flowfield and the otherwise (steady-state) stalled condition.

\section{Fluid Dynamics of an Airfoil's Impulsive Start}

Results for the lift and drag history of the airfoil, immediately following the impulsive start, are presented in Fig. 2. From the fluid dynamic point of view four different flow regions can be identified. These regions follow sequentially and their approximate duration can be identified by the following time intervals (so overlapping or transition between two intervals is possible):

Interval 1: $0.0<U_{\infty} t / c<0.02$ where the flow is attached but the streamlines are not yet parallel to the trailing edge.

Interval 2: $0.02<U_{\infty} t / c<2.0$ where the flow is attached and lift and streamline shapes follow the trends of ideal-flow calculations.

Interval 3: $2.0<U_{\infty} t / c<5.4$ where a trapped vortex develops above the airfoil, resulting in increased lift.

Interval 4: $5.4<U_{\infty} t / c<\infty$ where the flow is separated and accompanied by a periodic vortex shedding.

The computation of this flowfield provides a large body of information at each discrete time step, consisting of the velocity components and the pressure at each grid point. For the sake of brevity, only a representative set of information is presented on each interval. Thus, the discussion that follows focuses on the prominent changes in the airfoil's aerodynamics within each interval, with most explanations supported by numerical flow visualizations.

\section{Discussion of Flow Interval 1}

In terms of the airfoil's motion, the first flow interval can be further divided into two subintervals. The first one includes the initial impulse, whereas the second subinterval includes the time frame from the moment that the airfoil has reached its terminal speed and onward. With ideal fluid motion in mind, if the duration of the initial impulse was zero, then the acceleration was infinite, and the lift is also infinite (the sharply shooting upward lift curve in

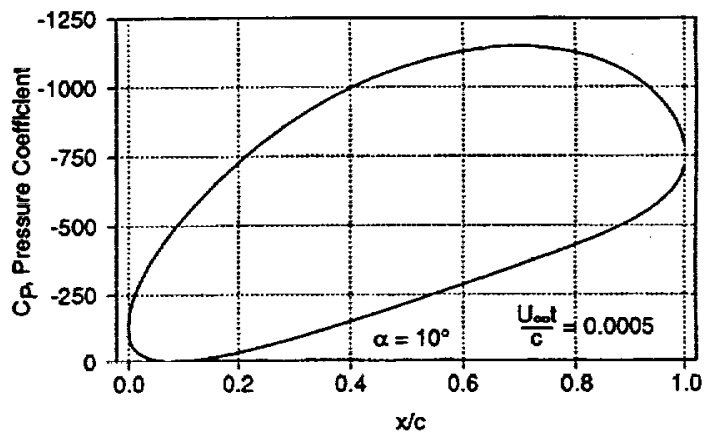

Fig. 3 Pressure coefficient along the airfoil's upper and lower surfaces (upper and lower curves, respectively) at the end of the initial acceleration.

Fig. 2 is hidden behind the ordinate). However, in this numerical computation the airfoil was assumed to be stationary at $U_{\infty} t / c=0$ and moving forward at speed of $U_{\infty}$ after the first time step. Thus, the forward acceleration was finite, and the loads on the airfoil during this initial acceleration resemble an elliptic loading. This is verified by the chordwise pressure distribution, depicted in Fig. 3, representing the condition at the end of the first time step. The large values of the pressure coefficient are a result of the small time step (rapid acceleration), but they are close to the values predicted by simple added-mass models (such as Eq. 13.40 from Ref. 8, yielding a midchord pressure difference of $\Delta C_{p} \approx 700$ for this case).

The rest of the first flow interval (excluding the initial acceleration) relates to the condition where the airfoil has reached its terminal speed and the flow is adjusting to this new condition. Because of the relatively short duration of the first interval, compared with the other three, the computations were rerun with a finer time step of $U_{\infty} \Delta t / c=0.0005$. Details of this calculation are shown by the instantaneous particle traces plotted in Fig. 4. The most interesting fluid dynamic aspect here is the flow around the trailing edge and the fact that the flow is attached along the rest of the airfoil's surface. Potential flow models, widely used for both steady and unsteady airfoil theory (Refs. 1-3), apply the so called Kutta condition. This condition, in general, requires that the flow leaves parallel to the airfoil's trailing edge (not as shown in the first frames of Fig. 4). Early flow visualizations (Ref. 19, pp. 394-397), however, demonstrated that initially a condition similar to the one shown in the first frame of Fig. 4 exists where there is flow around the trailing edge and a rear stagnation point exists on the upper surface of the airfoil. Therefore, the first question that comes to mind relates to the length of this initial condition. Based on the sequence presented in Fig. 4, initial signs showing the termination of this flow around the trailing edge are present as early as $U_{\infty} t / c=0.005$. At this point the starting vortex, with counterclockwise circulation, is clearly visible and it lifts off within the time frame $U_{\infty} t / c=0.01-0.02$. Throughout this initial process the flow on the airfoil is attached, but because of the flow around the trailing edge a suction peak develops there. Figure 5 shows the pressure distribution at $U_{\infty} t / c=0.005$ on the airfoil and the large suction peak at the trailing edge due to the flow around the trailing edge. Beyond $U_{\infty} t / c=0.02$ the flow leaves parallel to the trailing edge and the Kutta condition is satisfied.

Another important issue, relevant to the periodic unsteady (and attached) flow over airfoils, is the range of reduced frequencies, $\sigma=\omega c / 2 U_{\infty}$, for which the validity of the Kutta condition can be assumed. Studies (aimed at higher Reynolds numbers) such as Refs. 20 and 21, place the limit on the maximum frequency near $\sigma=2$, above which the Kutta condition cannot be applied. The present calculation can also contribute to the interpretation of this limit by identifying the time delay associated with the flow near the trailing edge. The numerical (attached) flow visualization within interval 1, presented in Fig. 4, implies that the trailing-edge flow adjusts to the form assumed by the Kutta condition within approximately $U_{\infty} t / c=0.02$. (Based on this number one can speculate that if a sinusoidal motion is replaced by, say, 100 impulsive motions per cycle, then the resulting reduced frequency is $\sigma=\pi / 2$, which is close to the experimental observation in Ref. 21.) 


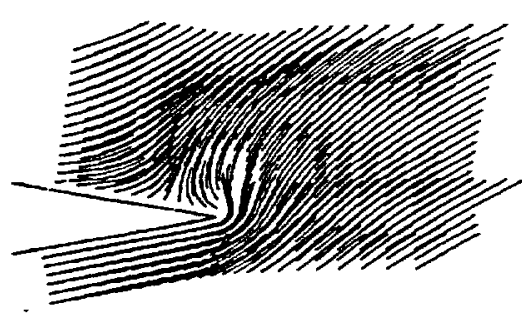

$\frac{U_{\infty \mathrm{ot}}}{\mathrm{c}}=0.0005$

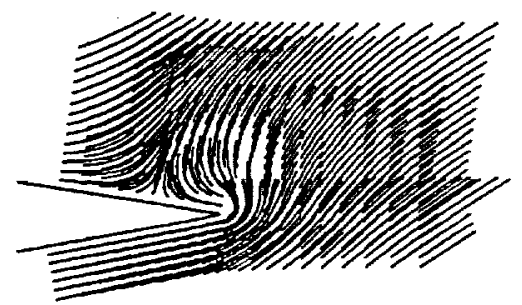

$\frac{U_{\text {oot }}}{\mathrm{c}}=0.0010$

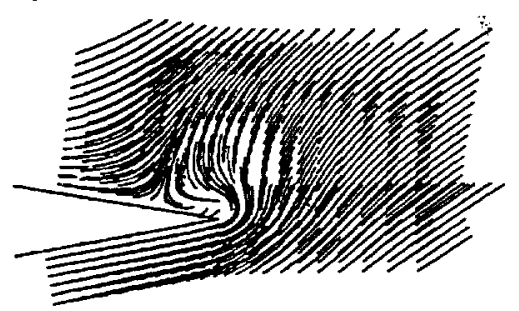

$\frac{U_{\text {oot }}}{c}=0.0015$

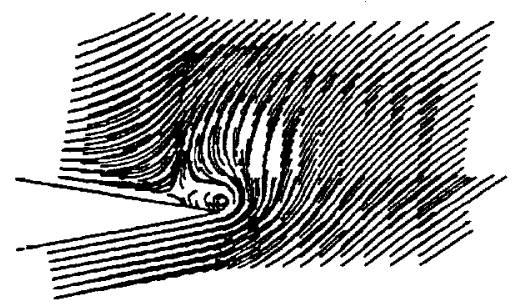

$\frac{U_{\infty} t}{C}=0.0020$

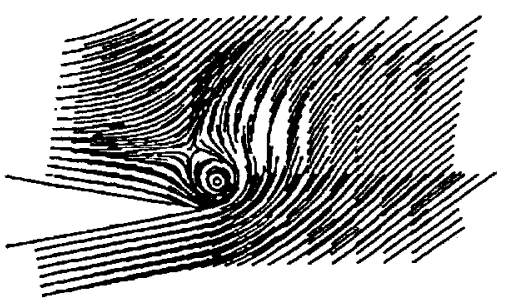

$\frac{U_{\infty \infty} t}{c}=0.0050$

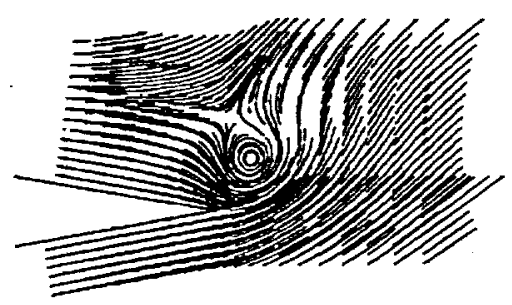

$\frac{U_{\text {ott }}}{c}=0.0100$

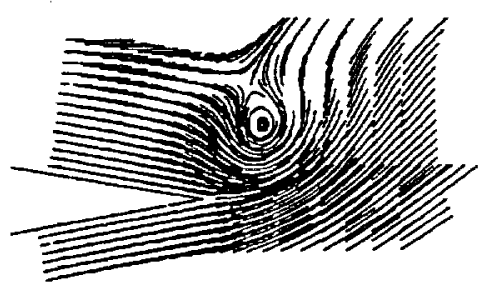

$\frac{U_{\infty} t}{c}=0.0150$

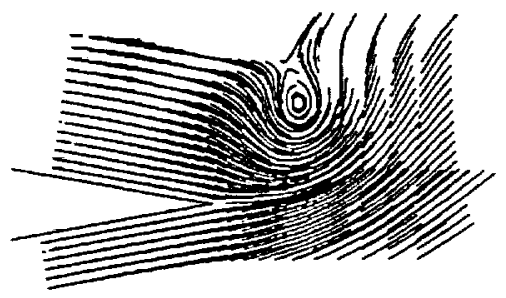

$\frac{U_{\infty} t}{c}=0.0200$

Fig. 4 Instantaneous particle paths of the flow near the airfoils trailing edge immediately after the impulsive start; $\alpha=10$ deg.

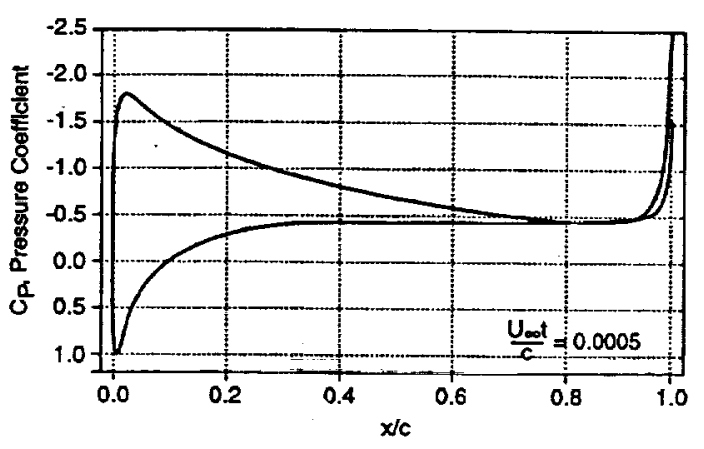

Fig. 5 Pressure coefficient distribution along the airfoil's upper and lower surfaces at $U_{\infty} l / c=0.005$ (interval 1).

\section{Discussion of Flow Interval 2}

Within this interval $\left(0.02<U_{\infty} t / c<2.0\right)$ the flow is attached, and both the streamline shapes and the pressure field follow closely the results of potential flow theory (e.g., Ref. 8, Chapter 13). This can be demonstrated by replotting the initial portion of Fig. 2 and by comparing the airfoil lift with potential flow results, as depicted in Fig. 6. The classical potential-flow values for the lift in Fig. 6 (Ref. 1) are very close to the present viscous calculations up to
$U_{\infty} t / c \approx 2$. Here the variation of the lift coefficient is controlled by the starting vortex that appeared at the end of the first interval. The downwash induced by this vorex is reducing the airfoil's lift (from the thin-airfoil theory, steady-state value of $C_{l}=2 \pi \alpha=1.097$ ). As the airfoil moves forward, leaving the strong starting vortex behind, the lift increases gradually. This sequence continues until the end of interval 2, at about $U_{\infty} t / c=2$, where a small trailing-edge separation begins to develop. The effect of the starting vortex is to increase the component of the pressure drag (resulting from the integration of the pressure distribution, see Ref. 3), immediately following the inpulsive start. This effect, which gradually diminishes, is also seen in the viscous calculations within the time interval $0<U_{\infty} t / c<0.5$. However, the contribution of the viscous drag is much larger and the effect is seen only as a small drag increase at the beginning of the motion. The airfoil drag during the rest of this interval can be attributed to the viscous boundary layer (since the steady-state ideal pressure integral over the airfoil yields zero drag).

A typical vorticity plot for the flow over the aiffoil in the middle of interval 2 (at $U_{\infty} t / c=1.0$ ) is presented in Fig. 7. This figure shows the attached flow condition and that vorticity is generated in the boundary layer near the airfoil's surface only. Vorticity is being shed into the wake and fragments of the strong starting vortex are still visible at the right-side end of the computational domain. The pressure distribution over the airfoil, at the same moment, is 


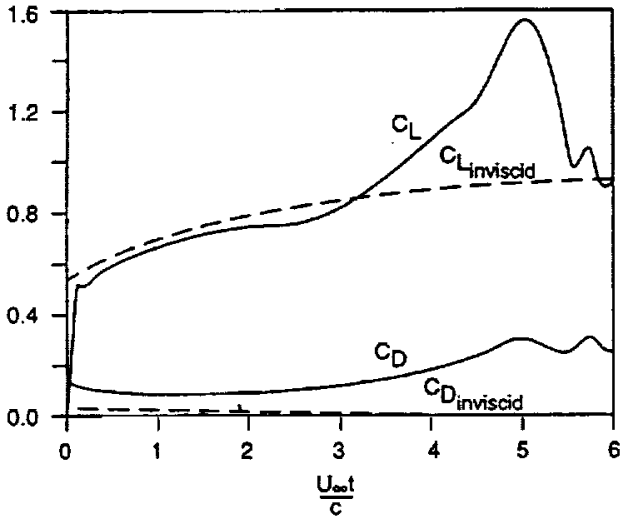

Fig. 6 Initial lift and drag coefficient transients after the impulsive forward motion of the NACA 0015 airfoil (solid line = Navier-Stokes computation and broken line $=$ potential-flow model).

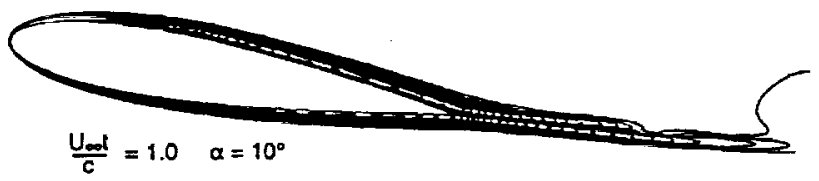

Fig. 7 Vorticity contour plot for the flow near the airfoil at $U_{\infty} t / c=$ 1.0. Note the residues of the starting vortex at the right-hand side of the computational domain.

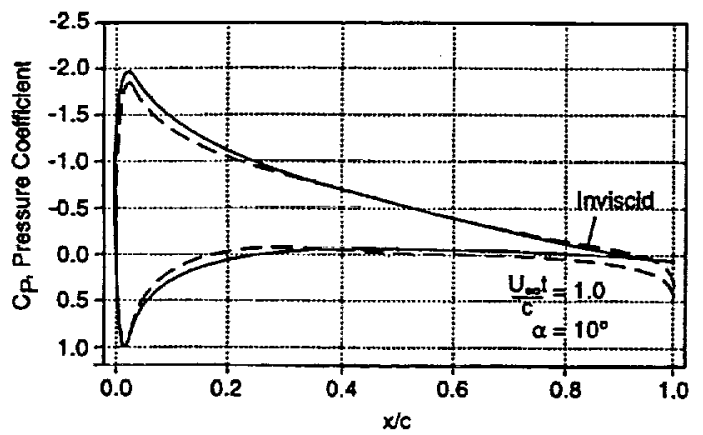

Fig. 8 Comparison between the viscons and potential fiow calculations for the pressure distribution over the airfoil at $U_{\infty} t / c=1.0$. The potential calculation is based on the panel method described in Ref. 8 , pp. 598-600.

presented in Fig. 8. Here comparison is made with a potential flow calculation and results of both methods seem to be very close. The viscous calculations, however, show a slightly lower lift generation towards the trailing edge, which is a result of the thickening boundary layer on the upper surface. This close agreement between the two methods and the flowfield description in Fig. 7 clearly indicate the attached flow condition prevailing in interval 2. This also indicates that the delay in the flow separation is on the order of $U_{\infty} \Delta t=2$ chord lengths, and the flow in the vicinity of the trailing edge, within interval 2 , is in accord with the Kutta condition.

\section{Discussion of Flow Interval 3}

Within this third interval a transition occurs between the attached and the separated flow conditions. However, this interval differs from the time-dependent separated flow in interval 4, primarily because of a large trapped vortex that considerably increases the lift of the airfoil. The large overshoot in the lift is clearly visible in Fig. 6 with a peak lift coefficient of approximately 1.6 , which is much larger than the potential-theory value of $C_{L}=1.097$, calculated for the steady-state case. This interval also demonstrates two important features, namely, the delay in the airfoil's (lift) stall and the potential to generate higher lift coefficients in unsteady flows. This can probably help to explain the large lift coefficients seen during dynamic heaving and flapping motions of wings (e.g., Refs. 22 and 23). A similar increase in the lift coefficient was observed during many of the early $y^{24}$ and more recent ${ }^{25.26}$ dynamic stall experiments (even though these were higher Reynolds number experiments).

The transition from the attached fowfield into the one with the trapped vortex is visualized by the sequence of vorticity plots in Fig. 9 (only the most significant frames are presented). As early as $U_{\infty} t / c=2.0$, the boundary layer thickens on the airfoil's upper surface and a small separation bubble emerges that becomes quite noticeable at $U_{\infty} t / c=3.0$ (Fig. 9, first frame). The shear layer originating close to the leading edge feeds this vortex, which gradually grows and moves backward (Fig. 9, $U_{\infty} t / c=4.0$ ). At about $U_{\infty} t / c=5.0$, an instability in the feeding shear layer develops, beginning the creation of a second such vortex. This second vortex becomes visible toward the end of this interval, at $U_{\infty} t / c=5.4$. During this time frame, a secondary vortex develops under the large vortex with opposite vorticity. The accumulation of these vortices eventually leads to the liftoff of the rear vortex and to the development of the periodic vortex shedding in interval 4. At the beginning of this interval the lower surface fow near the trailing edge seems to leave parallel to the surface. However, towards the end of this sequence, at $U_{\infty} t / c=5.6$, the flow moves around the trailing edge, creating a small and opposite vortex.

The large effect of the aforementioned trapped vortex on the airfoil's pressure distribution is shown in Fig. 10. Here two frames seem to be sufficient to visualize the process causing the increased lift. The first frame in Fig. 10 shows the pressures at $U_{\infty} t / c=4$, at a moment when the trapped vortex becomes clearly visible. The suction induced by this vortex on the upper surface seems to be the cause for the rise in the lift (and drag in Fig. 6). The second frame shows the pressure distribution when the secondary vortex signature
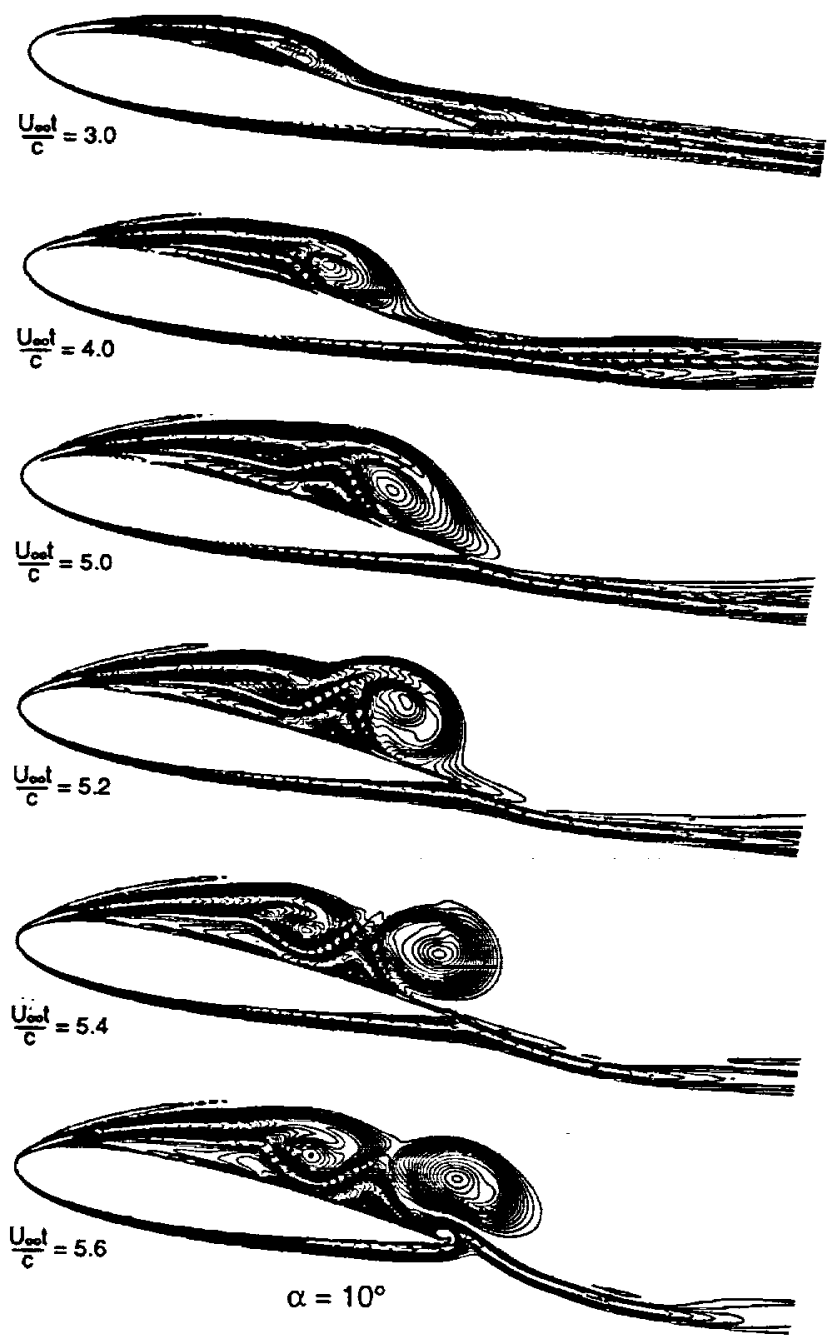

Fig. 9 Sequence of vorticity contour plots showing the development of the trapped vortex above the impulsively started airfoil. 

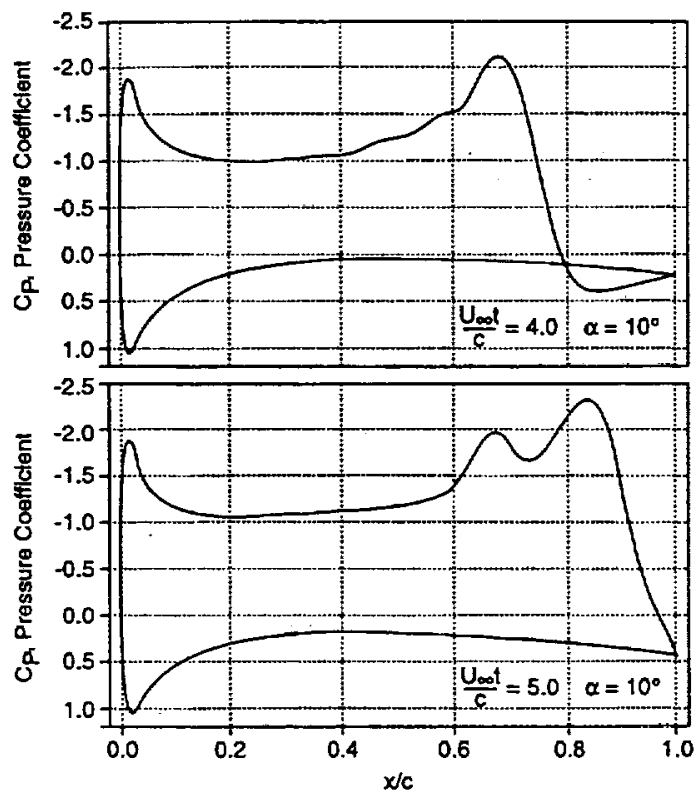

Fig. 10 Effect of the trapped vortex on the pressure distribution over the airfoil within flow interval 3.

becomes quite large, resulting in the highest lift coefficient in Fig. 6 . In the time that follows within interval 3 , these vortices move away from the airfoil, and the suction peaks and resulting lift is reduced. Finally, as shown by Fig. 9 , at $U_{\infty} t / c=5.6$, the leading-edge shear layer develops a wavy instability that leads to the periodic vortex shedding in interval 4.

\section{Discussion of Flow Interval 4}

In this interval the transient effect of the impulsive start has diminished and the well-known periodic vortex shedding prevails (see Fig. 2). The large-scale events during a vortex shedding cycle can be visualized by a sequence of vorticity plots, as shown in Fig. 11 . Locations of the large vortex structures are quite close to those observed during water-tunnel flow visualizations, at the same Reynolds number as the computations. This is seen in the photograph in Fig. 12, which compares well with the frame at $U_{\infty} t / c=25.5$, in Fig. 11. The streak lines in the photograph were visualized by injecting colored dye at the surface of the airfoil. To complement the discussion on the periodic vortex shedding process, the lift and drag coefficients were replotted in Fig. 13, for the corresponding time interval: $23.5<U_{\infty} t / c<26.5$. The shape of the lift and drag curves are quite similar, but a small delay between the two lines is visible. The delay may be caused by the reversed flow on the upper surface (during the low-drag moment, $U_{\infty} t / c=26.5$ ) that is reducing the skin-friction drag.

The vortex shedding sequence in Fig. 11 begins with the formation of the counter-rotating vortex at the trailing edge $\left(U_{\infty} t / c=25.1\right)$. The shear layer originating at the front begins to roll up as well. These two counter-rotating vortices induce downwash between them, causing a dip in the pressure distribution at the suction side of the airfoil (Fig. 14a). This condition makes up the low lift part of the cycle, as shown in Fig. 13. As the trailing-edge vortex size increases, it lifts off at $U_{\infty} t / c=25.5$, and the leading-edge vortex grows, increasing the airfoil's lift. At $U_{\infty} t / c=25.7$ the leading-edge vortex is the largest, whereas the trailing-edge vortex has already merged into the flow behind the airfoil. This is the highest lift condition, which continues until $U_{\infty} t / c=26.1$, when the clockwise rotating vortex lifts off. The corresponding pressure distribution is given in Fig. 14b, where the suction peak due to this vortex is clearly visible. As this vortex drifts with the flow, the trailing-edge vortex formation begins and the whole cycle is repeated. The calculated frequency of the periodic vortex shedding yields a Strouhal number of about $S_{t}=f d / U_{\infty}=0.15$ where $d$ is the airfoil's frontal height. This is quite close to the expected frequency at this condition and to the results of the flow visualizations.
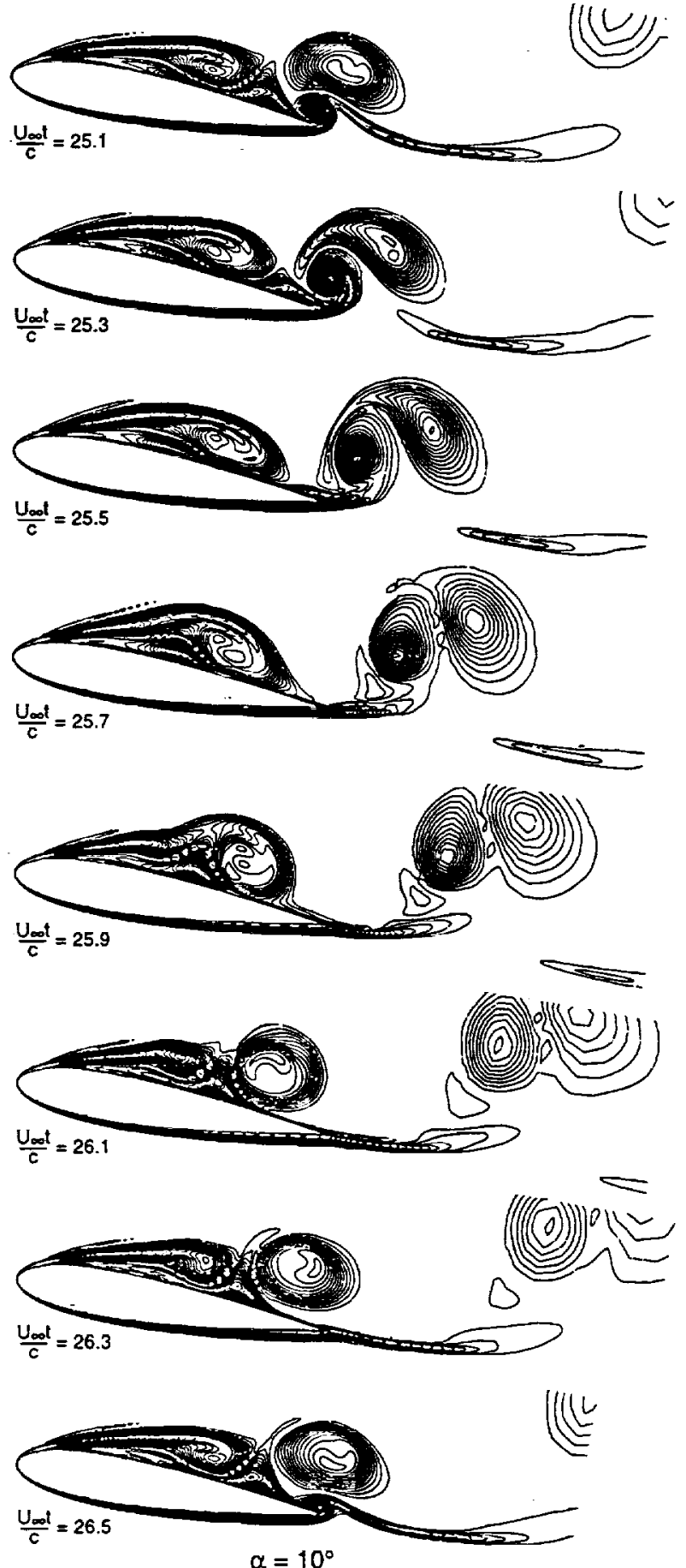

Fig. 11 Sequence of vorticity contour plots showing the periodic wake shedding process.

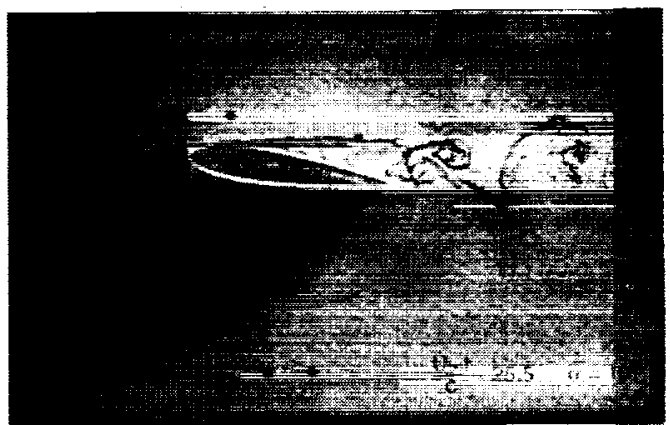

Fig. 12 Visualization of the flow over the NACA 0015 airfoil by colored dye in a water-tunnel experiment at a Reynold number of $1.2 \times 10^{4}$. 


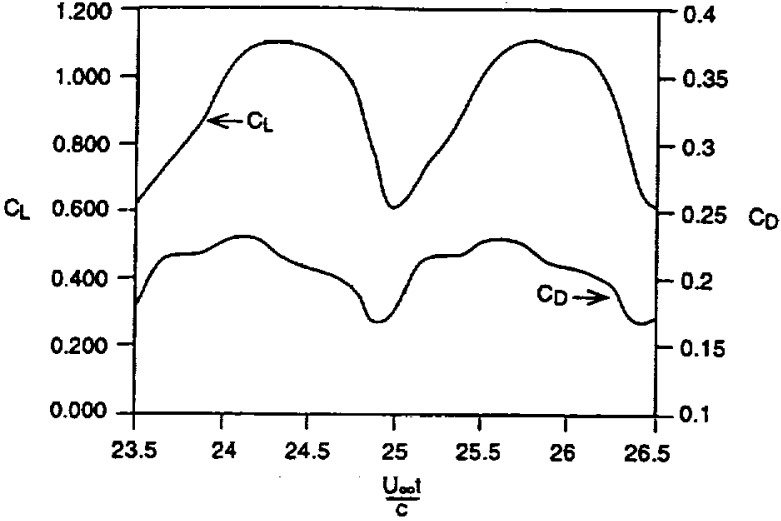

Fig. 13 Calculated lift and drag coefficient variation during the periodic wake shedding cycle.
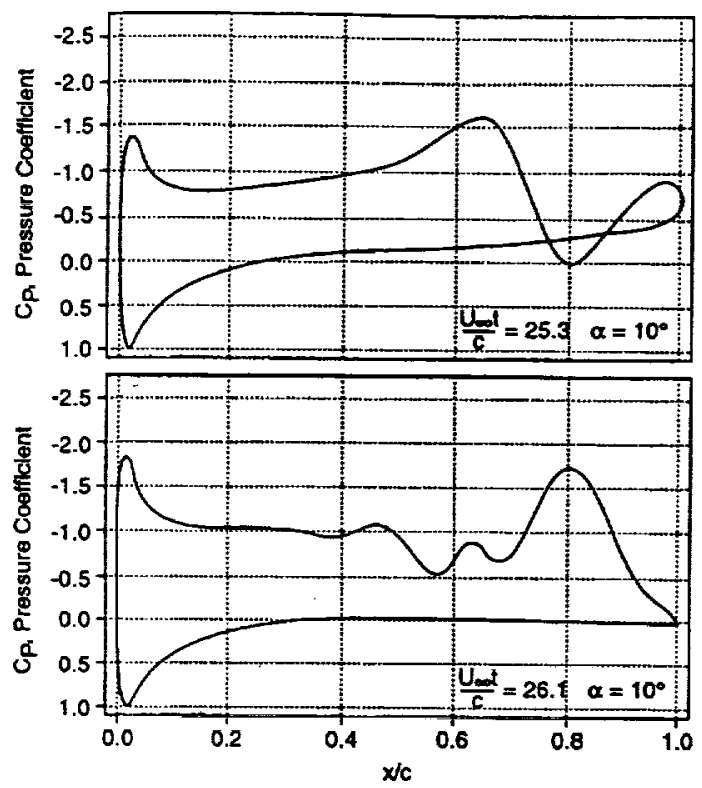

Fig. 14 Pressure coefficient distribution along the airfoils upper and lower surfaces, for the low and high lift conditions, during the wake shedding cycle.

\section{Concluding Remarks}

The fluid dynamic phenomena following the impulsive start of an airfoil include several sequential intervals, revealing numerous important features relevant to other unsteady flows. For example, the delay in the buildup of the trailing-edge flow (the Kutta condition) following the initial impulse lasts about $U_{\infty} \Delta t / c=0.02$. This information is important for understanding the frequency limits in certain periodic flows and in their mathematical models. Also, the computations demonstrate that there is a large delay, close to $U_{\infty} \Delta t / c=5$, in the buildup of the periodic, unsteady separated flow over a nonpitching airfoil. Furthermore, within this delayed period the airfoil's lift considerably outgrows its steady-state timeaveraged value, which fact may explain the large lift coefficients observed during flapping flight. This is caused by the large, initially trapped vortex, which is clearly identified by the numerical flow visualizations. At the end of this transient condition the trapped vortex leaves the aiffoil and the well-known periodic vortex shedding pattem prevails. The preceding fluid dynamics sequence resembles somewhat the flow features observed during dynamic stall studies on oscillating airfoils (e.g., Ref. 24). However, to the best knowledge of the authors, this is the first detailed examination of the stall sequence during an impulsive start.

\section{Acknowledgments}

This study was supported by NASA Ames Research Center Consortium agreement NCA2-786, with James Ross as project monitor.

\section{References}

'Wagner, H., "Uber die Entstehung des Dynamischen Autriebes von Tragflugeln," Zeitschrifi für Angewandte Mathematik und Mechanik, Vol. 5, No. 1, 1925, pp. 17-35.

${ }^{2}$ Katz, J., and Weihs, D., "The Effect of Chordwise Flexibility on the Lif of a Rapidly Accelerated Airfoil," Aeronautical Quarterly, Vol. 30, Pt. 1, 1979, pp. 360-369.

${ }^{3}$ Weihs, D., and Katz, J., "Transient Induced Drag," AIAA Journal, Vol. 24, No. 7, 1986, pp. 1203-1205.

${ }^{4}$ Chow, C. Y., and Huang. M. K., "The Initial Lift and Drag of an Impulsively Started Airfoil of Finite Thickness," Journal of Fluid Mechanics, Vol. 118, May 1982, pp. 393-409.

${ }^{5}$ Graham, J. M. R., "The Lift on an Airfoil in Starting Flow," Journal of Fluid Mechanics, Vol. 133, Aug. 1983, pp. 413-425.

${ }^{6} \mathrm{Katz}, \mathrm{J}$. , "A Discrete Vortex Method for the Non-Steady Separated Flow Over an Airfoil," Joumal of Fluid Mechanics, Vol. 102, Jan. 1981, pp. 315-328.

${ }^{7}$ Katz, J., "Calculation of the Aerodynamic Forces on Automotive Lifting Surfaces," Journal of Fluids Engineering, Vol. 107, No. 4, 1985, pp. 438-443.

${ }^{8}$ Katz, J., and Plotkin, A., Low-Speed Aerodynamics: From Wing Theory to Panel Methods, McGraw-Hill, New York, 1991, Chap. 13.

${ }^{9}$ Falco, R. E., Chu, C. C., Hetherington, M. H., and Gendrich, C. P., "The Circulation of an Airfoil Starting Vortex Obtaining from Instantaneous Vorticity Measurements Over an Area," AIAA Paper 88-3620, July 1988.

${ }^{10}$ Aihara, Y., Koyama, H., and Murashige, A., "Transient Aerodynamic Characteristics of a Two-Dimensional Airfoil During Stepwise Incidence Variation," Joumal of Aircraft, Vol. 22, No. 8, 1985, pp. $661-668$

"Sawyer, R. S., and Sullivan, J. P., "Lift Response of a Rectangular Wing Undergoing a Step Change in Forward Speed," AIAA Journal, Vol. 28, No. 7, 1990, pp. 1306, 1307.

${ }^{12}$ Finaish, F., and Frigerio, J., "Primary and Secondary Vortex Structures over Accelerated-Decelerated Airfoils at High Angles of Attack," SAE Paper 93-1368, Dayton, OH, April 1993.

${ }^{13}$ Rogers, S. E., and Kwak, D., "An Upwind Differencing Scheme for the Steady-State Incompressible Navier-Stokes Equations,"NASA TM 101051, Nov. 1988; see also Joumal of Applied Numerical Mathematics, Vol. 8, No. 1, 1991, pp. 43-64.

${ }^{14}$ Rogers, S. E., and Kwak, D., "An Upwind Differencing Scheme for the Time Accurate Incompressible Navier-Stokes Equations," AIAA Jourmal, Vol. 28, No. 2, 1990, pp. 253-262.

${ }^{15}$ Rogers, S. E., "Comparison of Implicit Schemes for the Incompressible Navier-Stokes Equations and Artificial Compressibility," AIAA Journal, Vol. 33, No. 11, 1995, pp. 2066-2072.

${ }^{16}$ Rogers, S. E., Wiltberger, N. L., and Kwak, D., "Efficient Simulation of Incompressible Viscous Flow over Single and Multi-Element Airfoils," AIAA Paper 92-0405, Jan. 1992; see also Journal of Aircraft, Vol. 30, No. 5, 1993, pp. 736-743.

${ }^{17}$ Rogers, S. E., "Progress in High-Lift Aerodynamic Calculations," Journal of Aircraft, Vol. 31, No. 6, 1994, pp. 1244-1251.

${ }^{18}$ Kiris, C., Rogers, S. E., Kwak, D., and Lee, Y. T., "TimeAccurate Incompressible Navier-Stokes Computations with Overlapping Moving Grids," Advances in Computational Methods in Fluid Dynam. ics, FED-Vol. 196, American Society of Mechanical Engineers, 1994, pp. 67-76.

${ }^{19}$ Karamchet, K., Principles of Ideal-Fluid Aerodynamics, Krieger. Malabar, FL, 1980

${ }^{20} \mathrm{Katz}$, J., and Weihs, D., "Wake Rollup and the Kutta Condition for Airfoils Oscillating at High Frequency," AIAA Journal, Vol. 19, No. 12, 1981, Pp. 1604-1606.

${ }^{21}$ Poling, D. R., and Telionis, D. P., "The Response of Airfoils to Periodic Disturbances-The Unsteady Kutta Condition," AlAA Journal, Vol. 24, No. 2, 1986, pp. 193-199

${ }^{22}$ Spedding, G. R., and Maxworthy, T., "The Generation of Circulation and Lift in a Rigid Two-Dimensional Fling," Journal of Fluid Mechanics, Vol. 165, April 1986, pp. 247-272.

${ }^{23}$ Sohn, M. H., and Wu, J. C., "A Numerical Study of the Weiss-Fogh Mechanism," AIAA Paper 87-0238, Jan. 1987.

${ }^{24}$ McCroskey, W. J., Car, L. W., and McAlister, K. W. "Dynamic Stall Experiments on Oscillating Airfoils," AIAA Paper 75-125, Jan. 1975.

${ }^{25}$ Robinson, M. C., and Wissler, J. B., "Unsteady Surface Pressure Measurements on a Pitching Rectangular Wing," AIAA Paper 88-0328, Jan. 1988.

${ }^{26}$ Schreck, S. J., Addington, G. A., and Luttges, M. W., "Flow Structure and Development Near the Root of a Straight Wing Pitching at Constant Rate," AIAA Paper 91-1793, June 1991. 